\title{
MSK1 downregulation is involved in inflammatory responses following subarachnoid hemorrhage in rats
}

\author{
BO NING ${ }^{1,2^{*}}$, ZHEN LI $^{1,3^{*}}$, LEI NING $^{4}$, JUN WU $^{5}$, XIN CHEN $^{5}$, \\ PENGJUN JIANG ${ }^{5}$, FUXIN LIN $^{6}$ and BING ZHAO
}

\author{
${ }^{1}$ Department of Neurosurgery, Shandong Provincial Hospital, Shandong University, Jinan, Shandong 250021; \\ ${ }^{2}$ Department of Neurosurgery, Guangzhou Red Cross Hospital, Jinan University, Guangzhou, Guangdong 510220; \\ ${ }^{3}$ Department of Neurosurgery, Tai'an Central Hospital, Taishan Medical University, Tai'an, Shandong 271000; \\ ${ }^{4}$ Department of Medical Records, Affiliated Hospital of Taishan Medical University, Taishan Medical University, \\ Tai'an, Shandong 271000; ${ }^{5}$ Department of Neurosurgery, Beijing Tiantan Hospital, Capital Medical University, \\ Beijing 100050; ${ }^{6}$ Department of Neurosurgery, The First Affiliated Hospital of Fujian Medical University, \\ Fujian Medical University, Fuzhou, Fujian 350005; ${ }^{7}$ Department of Neurosurgery, Ren Ji Hospital, \\ School of Medicine, Shanghai Jiao Tong University, Shanghai 200127, P.R. China
}

Received February 15, 2019; Accepted January 2, 2020

DOI: $10.3892 / \mathrm{etm} .2021 .9795$

\begin{abstract}
Subarachnoid hemorrhage (SAH) is a life-threatening neurological disease. Recently, inflammatory factors have been confirmed to be responsible for the brain damage associated with SAH. Therefore, studying the post-SAH inflammatory reaction may clarify the mechanism of SAH.Mitogen and stress-activated protein kinase 1 (MSK1) causes the phosphorylation of NF- $\mathrm{KB}$ and regulates the activity of pro-inflammatory transcription factors. The present study aimed to identify the potential role of MSK1 in inflammation and brain damage development following SAH. A cisterna magna blood injection model was established in Sprague-Dawley rats. Hematoxylin and eosin staining, reverse transcription-quantitative polymerase chain reaction assays and double immunofluorescence staining were used to analyze the role of MSK1, IL-1 $\beta$ and TNF- $\alpha$ in the inflammatory process after SAH. In a model of lipopolysaccharide-induced astrocyte inflammation, the effect of overexpressing MSK1 overexpression was analyzed by western blot analysis. The results demonstrated that MSK1
\end{abstract}

Correspondence to: Dr Fuxin Lin, Department of Neurosurgery, The First Affiliated Hospital of Fujian Medical University, Fujian Medical University, 20 Chazhong Road, Fuzhou, Fujian 350005, P.R. China

E-mail: 1fxstudy@126.com

Dr Bing Zhao, Department of Neurosurgery, Ren Ji Hospital, School of Medicine, Shanghai Jiao Tong University, 160 Pujian Road, Shanghai 200127, P.R. China

E-mail: bzns@163.com

"Contributed equally

Key words: subarachnoid hemorrhage, mitogen and stress-activated protein kinase 1, inflammation expression were negatively correlated with TNF- $\alpha$ and IL-1 $\beta$ expression levels, and reached peak levels 2 days after TNF- $\alpha$ and IL-1 $\beta$. The double immunofluorescence staining results showed that the expression of MSK1 was in the same plane of view as TNF- $\alpha$ and IL-1 $\beta$ in the brain cortex. Furthermore, the in vitro studies indicated that the overexpression of MSK1 inhibited the expression of TNF- $\alpha$ and IL- $1 \beta$ following LPS challenge. These results imply that MSK1 may be involved in the inflammatory reaction following SAH, and may potentially serve as a negative regulator of inflammation.

\section{Introduction}

Subarachnoid hemorrhage (SAH), primarily caused by a ruptured aneurysm, is a life-threatening neurological disease, accounting for 5\% of all stroke types (1). The incidence of SAH is $8-9$ per 100,000 individuals (2). Despite significant improvements in the diagnosis and surgical treatment of $\mathrm{SAH}$, $25 \%$ of patients succumb to the disease, and $50 \%$ of survivors suffer significant disabilities $(3,4)$.

Previous clinical and experimental evidence supports the hypothesis that inflammatory factors are the basis of vascular constriction and brain damage associated with SAH $(5,6)$. Jedrzejowska-Szypulka et al (7) observed that increased levels of interleukin-1 $\beta$ (IL-1 $\beta$ ) can cause nerve toxicity and brain edema by destroying the blood-brain barrier (BBB). A previous study revealed that IL-6 and IL-8 levels were increased following SAH (8). Furthermore, inhibition of tumor necrosis factor- $\alpha$ (TNF- $\alpha$ ) was demonstrated to decrease apoptosis in the hippocampus following SAH (9).

As inflammation is a key factor involved in the pathology of SAH, the identification of a novel cytokine involved in inflammation may aid in the detailed characterization of the mechanisms of SAH.

Mitogen and stress-activated protein kinase 1 (MSK1) is a nuclear protein kinase (10) that contains 2 kinase 
domains: A C-terminal kinase domain associated with the $\mathrm{Ca}^{2+} /$ calmodulin-dependent kinase family and an N-terminal kinase domain associated with the cAMP-dependent, cGMP-dependent and protein kinase $\mathrm{C}$ kinase family. It was originally identified through its homology to the N-terminal ribosomal S6 kinase domain (11). MSK1 is involved in the $\mathrm{cAMP} / \mathrm{cAMP}$ dependent protein kinase (PKA)/cAMP responsive element binding protein 1 (CREB) pathway and the activation of $\mathrm{NF}-\kappa \mathrm{B}$, which are key mediators in the transcription of genes involved in inflammatory responses (12). Several studies have demonstrated that MSK1 serves a positive role in the regulation of the activity of pro-inflammatory transcription factors implicated in asthma (13), and in vitro data suggest that MSK1 is involved in negative feedback pathways that are critical in preventing uncontrolled inflammation in macrophages (14). In addition, MSK1 may be implicated in the anti-inflammatory properties of glucocorticoids through its translocation from the nucleus to the cytoplasm (15).

Despite evidence of a correlation between MSK1 expression and regulation of inflammation, specific genetic evidence supporting the role of MSK1 in central nervous system (CNS) inflammation following SAH has not been reported to date.

Therefore, we hypothesized that MSK1 may be closely associated with inflammation during the process of SAH, and the present study aimed to identify the potential role of MSK1 in inflammation and subsequent brain damage development following $\mathrm{SAH}$.

\section{Materials and methods}

Animals and surgical procedures. In the animal model, a total of 60 Sprague-Dawley rats (age, 3 months; weight, 280-320 g) were obtained from the Shandong Experimental Animal Center. All animals were maintained on a 12-h light/dark cycle with ad libitum access to water and food and the animal room had a temperature of $20-25^{\circ} \mathrm{C}$ and a relative humidity of 45-50\%. The SAH model was established as described previously (9). In the study group, the death rate was 0.

The animals were anesthetized with intraperitoneal (IP) injection of $10 \%$ chloral hydrate $(400 \mathrm{mg} / \mathrm{kg})$. Then, the animals were placed in a stereotactic frame with the head tilted down at $\sim 30^{\circ}$. A midline scalp incision was made in the neck and the atlanto-occipital membrane was exposed upon separating the underlying muscle. Then, the membrane was punctured with a 27-gage needle, and $0.3 \mathrm{ml}$ autologous femoral arterial blood was injected into the cisterna magna within 10 min with an infusion pump. Animals in the sham group were injected with $0.3 \mathrm{ml}$ sterile saline. Rats had ad libitum access to water and food upon recovery from anesthesia. The animals with SAH were randomly divided into 7 subgroups and sacrificed by decapitation prior to anesthesia with $10 \%$ chloral hydrate (400 mg/kg; IP) at 2 h, 6 h, 12 h, day 1, day 3, day 5 and day 7 post-SAH $(n=6)$. Sham animals $(n=6)$ experienced the same surgery but without injection of blood into the cisterna magna. Consequently, the sham animals were sacrificed $24 \mathrm{~h}$ following the sham surgery. No signs of peritonitis such as decreased food intake and abdominal swelling were observed following the administration of $10 \%$ chloral hydrate. Death was verified using the following criteria: Confirmation of the absence of visible breathing and measurable heartbeat, confirmation of pupil dilation and absence of the tail pinch reflex. The animal use and care protocols, including all surgical procedures, were approved by the Animal Care and Use Committee of Taishan Medical University and conformed to the Guide for the Care and Use of Laboratory Animals by the National Institute of Health (16).

Hematoxylin and eosin (H\&E) staining. Microscope slides containing cryosections of tissue were fixed in $100 \%$ alcohol for 5 mins and then immersed in $\mathrm{H}_{2} \mathrm{O}$ for $30 \mathrm{sec}$ with manual agitation. The slides were placed in a Coplin jar containing Mayer's hematoxylin and agitated for $30 \mathrm{sec}$. Following rinsing of the slides in $\mathrm{H}_{2} \mathrm{O}$ for $1 \mathrm{~min}$ and staining with $1 \%$ eosin Y solution for 10-30 sec, the sections were dehydrated with 2 washes in $95 \%$ experimental ethanol and 2 washes in $100 \%$ experimental ethanol for $30 \mathrm{sec}$ each. The alcohol was extracted with 2 washes in xylene, and 1-2 drops of mounting medium were then added to the slides, which were covered with a coverslip. All steps were at room temperature. The stained slides were examined with a light microscope (Leica Microsystems, Inc.) at x100 and x400 magnification.

Reverse transcription-quantitative polymerase chain reaction (RT-qPCR). TRIzol ${ }^{\circledR}$ Reagent (Thermo Fisher Scientific, Inc.) was used to isolate total RNA from rat tissues, and the RNA concentration was determined by spectrophotometric analysis (optical density 260/280). cDNA was synthesized with Reverse Transcriptase reagent (Takara Biotechnology Co., Ltd.). RT-qPCR was performed using Stratagene Mx3000P qPCR system (Agilent Technologies, Inc.) in a reaction that contained $2 \mu \mathrm{l}$ cDNA, $12.5 \mu \mathrm{l}$ SYBR-Green (Takara Biotechnology Co., Ltd.), $1 \mu \mathrm{l}$ each forward and reverse primers $(10 \mu \mathrm{M})$ and RNase-free $\mathrm{H}_{2} \mathrm{O}$ to a final volume of $25 \mu \mathrm{l}$. The following primers were used: MSK1 forward (FW) 5'-CCTCAAGAC CCCATGCTTCA-3'; MSK1 reverse (REV) 5'-ACTTCTGTC ATGGGACTGGA-3'; TNF- $\alpha$ FW 5'-TGCCTATGTCTCAGC CTCTTC-3'; TNF- $\alpha$ REV 5'-GAGGCCATTTGGGAACTT CT-3'; IL-1 $\beta$ FW 5'-TGAGCACCTTCTTTTCCTTCA-3'; IL-1 $\beta$ REV 5'-TTGTCTAATGGGAACGTCACAC-3'; GAPDH FW 5'-GAGGCCGGTGCTGAGTATGT-3'; and GAPDH REV 5'-GGTGGCAGTGATGGCATGGA-3'. The thermocycling conditions used to perform the qPCR were as listed: Initial denaturation at $95^{\circ} \mathrm{C}$ for $30 \mathrm{sec}$, followed by 40 cycles of denaturation at $95^{\circ} \mathrm{C}$ for $5 \mathrm{sec}$ and annealing at $60^{\circ} \mathrm{C}$ for $30 \mathrm{sec}$. The expression levels of target genes were evaluated by using the $2^{-\Delta \Delta \mathrm{Cq}}$ method (17). All samples were analyzed in triplicate.

Double immunofluorescent staining. Immunofluorescent staining was performed according to our previous study (18). Brain tissue was fixed in $4 \%$ paraformaldehyde for $3 \mathrm{~h}$ at $4^{\circ} \mathrm{C}$ and then incubated in $20 \%$ saccharose for 2 days at $4{ }^{\circ} \mathrm{C}$, followed by incubation in $30 \%$ sucrose for an additional 2 days at $4^{\circ} \mathrm{C}$ to remove the $\mathrm{H}_{2} \mathrm{O}$ content. Sections measuring $8 \mu \mathrm{m}$ in thickness were prepared and blocked with $5 \%$ normal fetal bovine serum in PBS (Sigma-Aldrich; Merck KGaA) containing $0.1 \%$ Triton $\mathrm{X}-100$ for $2 \mathrm{~h}$ at room temperature. The slices were then incubated overnight at $4^{\circ} \mathrm{C}$ with primary antibodies against MSK1 (cat. no. ab81294; 1:200; Abcam), TNF- $\alpha$ (cat. no. ab13597; 1:200; Abcam), IL-1 $\beta$ (cat. no. ab9722; 
1:100; Abcam) and glial fibrillary acidic protein (GFAP; cat. no. sc7114; 1:100; Santa Cruz Biotechnology, Inc.). On the following day, the corresponding secondary antibodies (cat. nos. sc-2030 and sc-2354; 1:100; Santa Cruz Biotechnology, Inc.) were added and incubated for $2 \mathrm{~h}$ at room temperature in the dark. Following 3 washes in PBS, the slides were covered with a microscopic glass coverslip and observed under a Leica fluorescence microscope at x100 and x400 magnification (Leica Microsystems Inc.). Negative controls were prepared by omitting the primary antibodies.

Cell culture and treatment. Primary astrocytes were obtained from the cerebral cortex of 10 neonatal (2 day-old) male Sprague-Dawley rat pups obtained from Shandong Experimental Animal Center. The pups were decapitated and their brains were rapidly removed before the cerebral cortices were trypsinized and dissociated by trituration. The dissociation mixture was plated at a density of $5 \times 10^{7}$ cells per $75 \mathrm{~cm}^{2}$ flask in Dulbecco's modified Eagle's medium (DMEM) with F12 nutrient (1:1; Gibco; Thermo Fisher Scientific, Inc.). Cells were maintained in complete culture medium for 7-8 days. Prior to experimental treatments, cultures of astrocytes were passaged twice. The culture medium was exchanged for serum-free DMEM/F12 and experiments were initiated $24 \mathrm{~h}$ later. To assess the effect of lipopolysaccharide (LPS), cells were allowed to reach $80 \%$ confluence. At the time of treatment, $1 \mu \mathrm{g} / \mathrm{ml}$ LPS was added to the culture medium. Cells were stimulated with LPS for $24 \mathrm{~h}$. The reagents were administered directly to the growth medium for $48 \mathrm{~h}$. Non-treated cells were included as controls in all experiments.

Transfection. Astrocytes were seeded at a density of $2 \times 10^{5}$ cells $/ \mathrm{ml}$ in a 6 -well plate prior to transfection. After $24 \mathrm{~h}, 10 \mu \mathrm{l}$ Lipofectamine 2000 reagent in $450 \mu$ Opti-MEM $^{\circledR}$ (Invitrogen; Thermo Fisher Scientific, Inc.) and $500 \mu \mathrm{l}$ transfection mixture containing $4 \mathrm{mg}$ enhanced green fluorescent protein(EGFP)-N3-MSK1 plasmid (or EGFP-N3 mock plasmid as control) were added to each well. The cells were incubated for $48 \mathrm{~h}$ after transfection and then harvested for experiments. The experiments were repeated $\geq 3$ times. Plasmids were synthesized and donated by the Nerve Regeneration Laboratory of Nantong University. The EGFP-N3-MSK-1 contained the full sequence of MSK-1, while the control plasmid contained the insert 5'-TACAAGTAAAGCGGCCGCGACT-3'.

Western blot analysis. For western blot analysis, the cells were washed twice in ice-cold PBS and then homogenized in lysis buffer (1\% sodium deoxycholate, $50 \mathrm{mmol} / \mathrm{l}$ Tris, $1 \%$ NP-40 Lysis Buffer), $1 \%$ Triton X-100, 5 mmol/1 EDTA, 1\% SDS, $1 \mathrm{mmol} / 1$ phenylmethylsulfonyl fluoride $10 \mathrm{IU} / \mathrm{ml}$ aprotinin and $1 \mathrm{IU} / \mathrm{ml}$ leupeptin). The mixture was collected with a cell scraper. After centrifugation at $14,000 \mathrm{x}$ g for $15 \mathrm{~min}$ at $4^{\circ} \mathrm{C}$, the supernatant was collected. Then, a BCA kit (Thermo Fisher Scientific, Inc.) was used to determine the protein concentration. The samples were boiled for $5 \mathrm{~min}$ at $100^{\circ} \mathrm{C}$ upon adding SDS sample buffer. Samples ( $80 \mu \mathrm{g} / \mathrm{lane})$ were subjected to electrophoresis in 10\% SDS-PAGE gels for $40 \mathrm{~min}$ at $70 \mathrm{~V}$ followed by $90 \mathrm{~min}$ at $120 \mathrm{~V}$, and then transferred onto polyvinylidene difluoride membranes for $2.5 \mathrm{~h}$ at $180 \mathrm{~mA}$. The membranes were blocked with $5 \%$ non-fat milk for $2 \mathrm{~h}$ at room temperature and then incubated with the appropriate primary antibodies MSK1 (cat. no. ab81294; 1:200; Abcam), TNF- $\alpha$ (cat. no. ab13597;1:200; Abcam), IL-1 $\beta$ (cat. no. ab9722; 1:100; Abcam), $\beta$-actin (cat. no. sc-81178; 1:100; Santa Cruz Biotechnology, Inc.) at $4^{\circ} \mathrm{C}$ overnight. The membrane was then washed with PBS $+1 \%$ Tween-20 3 times and incubated with the appropriate horseradish peroxidase-conjugated secondary antibody (cat. no. A-16078; 1:2,000; Pierce; Thermo Fisher Scientific, Inc.) for $2 \mathrm{~h}$ at room temperature. The blotted protein bands were developed using ECL Chemiluminescent Substrate Reagent kit (Thermo Fisher Scientific, Inc.) and exposed to X-ray film. The films were estimated using a Molecular Dynamics densitometer (Scion Corporation). The relative protein expression levels were normalized to $\beta$-actin and quantified by Quantity One software (Version 4.6.6; Bio-Rad Laboratories, Inc.) through measuring the relative band density.

Statistical analysis. All values are expressed as means \pm standard error of the mean and each experiment was repeated $\geq 3$ times. SPSS v.21.0 (IBM Corp.) was used for statistical analysis of the data. Statistical evaluation was processed by two-tailed Student's t-test between two groups. Statistical evaluation of multiple groups was performed using a one-way analysis of variance followed by Dunnett's post hoc test. $\mathrm{P}<0.05$ was considered to indicate a statistically significant difference. The correlations between MSK1, IL- $1 \beta$ and TNF- $\alpha$ were determined by Spearman's analysis.

\section{Results}

Infiltration of inflammatory cells following SAH. Immunohistochemical staining was performed on cross-sections of adult rat brains. The number of inflammatory cells infiltrated in the day-1 group $\left(412.6 \pm 61.9\right.$ cells $\left./ \mathrm{mm}^{2} ; \mathrm{n}=6\right)$ was markedly elevated compared with the sham group $\left(113.0 \pm 47.5\right.$ cells $/ \mathrm{mm}^{2}$; $\mathrm{n}=6$ ). There was a statistically significant difference among the sham, the day- 1 and the day-3 groups (Figs. 1 and 2). The results revealed a high density of inflammatory cells (predominantly monocytic and neutrophilic granulocytes) in the cortex, which indicated that the inflammatory reaction appeared at an early stage following SAH.

Expression of MSK1,TNF- $\alpha$ and IL-1 $\beta$ during inflammatory responses following $S A H$. Although MSK1 is known to be expressed at relatively high levels in the nervous system, its function is well understood. In order to clarify whether MSK1 participates in the pro-inflammatory reaction during the process of SAH, the present study firstly examined the mRNA expression levels of MSK1, IL-1 $\beta$ and TNF- $\alpha$ in the brain cortex at various survival times following SAH. The quantitative analysis demonstrated high expression of MSK1 mRNA in the control and sham groups. Furthermore, the level of MSK1 in the cortex decreased gradually following SAH, and then peaked at day 3 post-SAH and increased during the following days. Conversely, quantitative analysis of the levels of IL- $1 \beta$ and TNF- $\alpha$ mRNA revealed that they were increased in the cortex as early as $2 \mathrm{~h}$ after SAH, reaching peak levels at day 1 after SAH and gradually decreasing during the subsequent days. MSK1 levels peaked 2 days later compared with those of IL- $1 \beta$ and TNF- $\alpha$. There was 


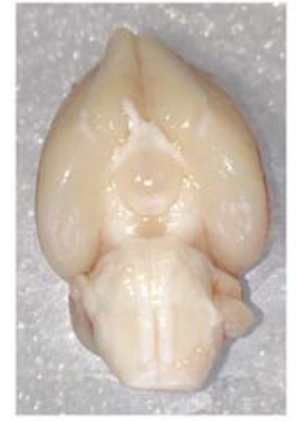

Sham

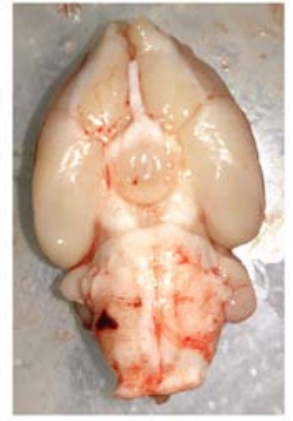

1Day
Figure 1. Animal model construction. Left image represents rat brain tissue from the sham group. The right image demonstrates rat brain tissue harvested 1 day after experimental subarachnoid hemorrhage.
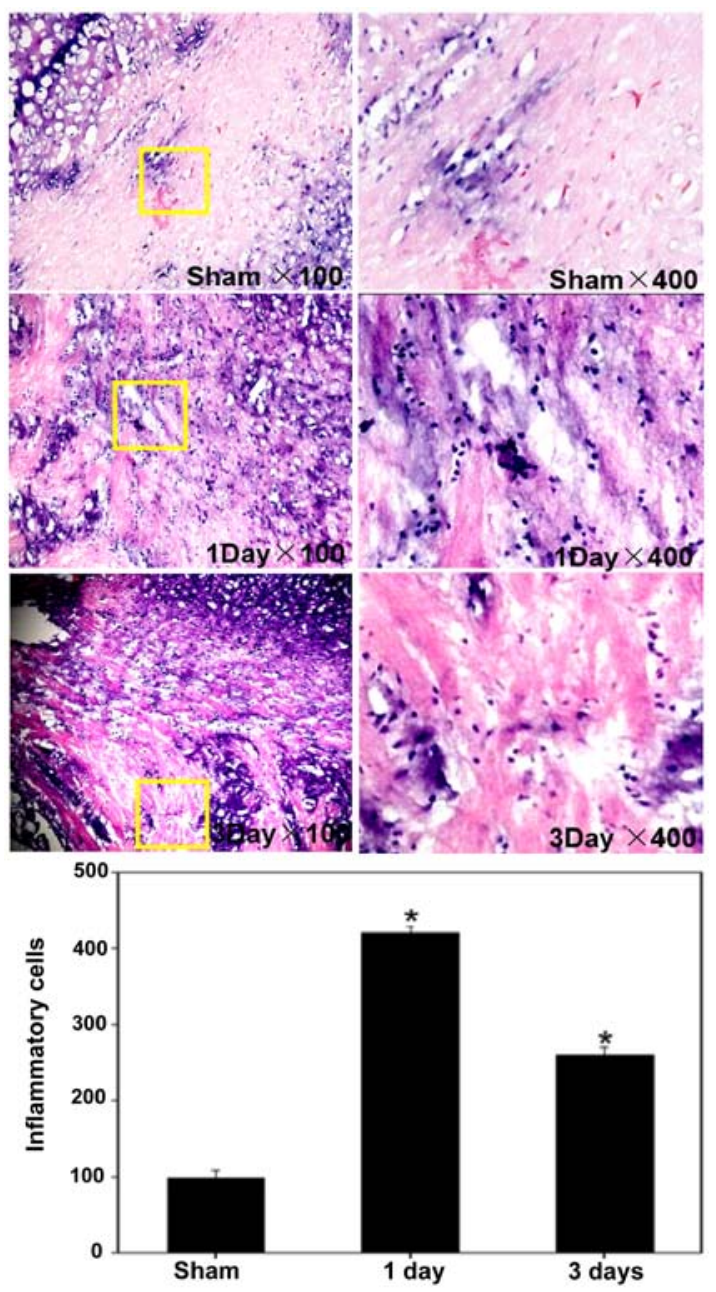

Figure 2. Hematoxylin and eosin staining of inflammatory cells in cross sections in adult rat brain tissues. Local infiltration by inflammatory cells (predominantly monocytic and a neutrophilic granulocyte) increased markedly in the brain tissues at 1 and 3 days after subarachnoid hemorrhage compared with the sham group. Statistical evaluation of the three groups was performed with a one-way analysis of variance followed by Dunnett's post hoc test. ${ }^{*} \mathrm{P}<0.05$ vs. sham group.

a marked positive correlation between IL-1 $\beta$ and TNF- $\alpha$ mRNA levels $(\mathrm{r}=0.741 ; \mathrm{P}<0.05)$. In addition, negative correlations were observed between MSK1 and IL-1 $\beta$ and TNF- $\alpha$ mRNA levels ( $r=-0.679$ and -0.709 , respectively; both $\mathrm{P}<0.05$; Fig. 3).
Co-localization of MSK1 with TNF- $\alpha, I L-1 \beta$ and GFAP. Double immunofluorescence staining for MSK1, TNF- $\alpha$, IL-1 $\beta$ and GFAP in the cortex was performed at day 1 after SAH to further explore the association between MAK1 and pro-inflammatory cytokines. Following injury, sections of adult rat brains were labeled with MSK1 and GFAP (an astrocyte marker), and co-localization of MSK1 with GFAP was observed in the brain tissue. Furthermore, immunofluorescent staining was performed, and the merged images revealed that MSK1 was located in the same plane of view in the brain cortex as TNF- $\alpha$ and IL-1 $\beta$ (Fig. 4).

MSK1 inhibits LPS-induced inflammation in cultured primary astrocytes. To additionally verify the aforementioned results, LPS was used to induce an inflammatory reaction in cultured primary astrocytes. It was observed that the TNF- $\alpha$ levels were significantly increased in the presence of minimum concentrations of LPS (1 $\mu \mathrm{g} / \mathrm{ml})$ (Fig. 5). To investigate whether MSK1 inhibits the expression of inflammatory factors in these cells, the cultured primary astrocytes were transfected with plasmids encoding MSK1. Western blot analysis was used to examine the levels of TNF- $\alpha$ in MSK1-transfected astrocytes in the presence of $1 \mu \mathrm{g} / \mathrm{ml}$ LPS. The results demonstrated that the overexpression of MSK1 decreased the expression of TNF- $\alpha$ (Fig. 6).

\section{Discussion}

SAH remains a devastating disease with high morbidity and mortality rates, and $50 \%$ of survivors suffer neurological dysfunctions that range from mild cognitive deficits to disabling cerebral infarctions. The majority of previous studies have focused on cerebral vasospasm (CVS), which is considered the major cause of cerebral ischemia and poor outcomes following SAH (19-21). However, clinical evidence suggests that certain patients deteriorate neurologically without accompanying vasospasm following SAH. In addition, preventing vasospasm does not always improves outcomes (22). The results of the CONSCIOUS-1 trial (trial no. 00111085), which investigated the role of clazosentan in preventing the occurrence of CVS following an aneurysmal SAH, also demonstrated that the pathophysiology underlying $\mathrm{SAH}$ is multifactorial and other pathological mechanisms independent of vasospasm may be responsible for poor clinical outcomes (23-25).

Both animal models and human studies have demonstrated that inflammation is an important factor following $\mathrm{SAH}$, inducing both direct brain injury and vasospasm, which in turn leads to brain ischemia $(5,26)$. An increased level of pro-inflammatory factors in the cerebrospinal fluid of patients with SAH is associated with poor outcomes $(27,28)$.

In the present study, H\&E staining demonstrated a high density of inflammatory cells in brain cortex 1 day after SAH. To evaluate the underlying role of inflammation, IL- $1 \beta$ and TNF- $\alpha$ were detected as inflammation-associated factors. IL-1 $\beta$, an important initiator of inflammation, signals through the IL-1 receptor to trigger multiple cellular responses. IL-1 $\beta$ is released from activated microglia upon brain inflammatory reaction $(7,9)$. TNF- $\alpha$ is another pro-inflammatory cytokine that can enhance vascular permeability, and serves a role in the recruitment of inflammatory cells and endothelial 

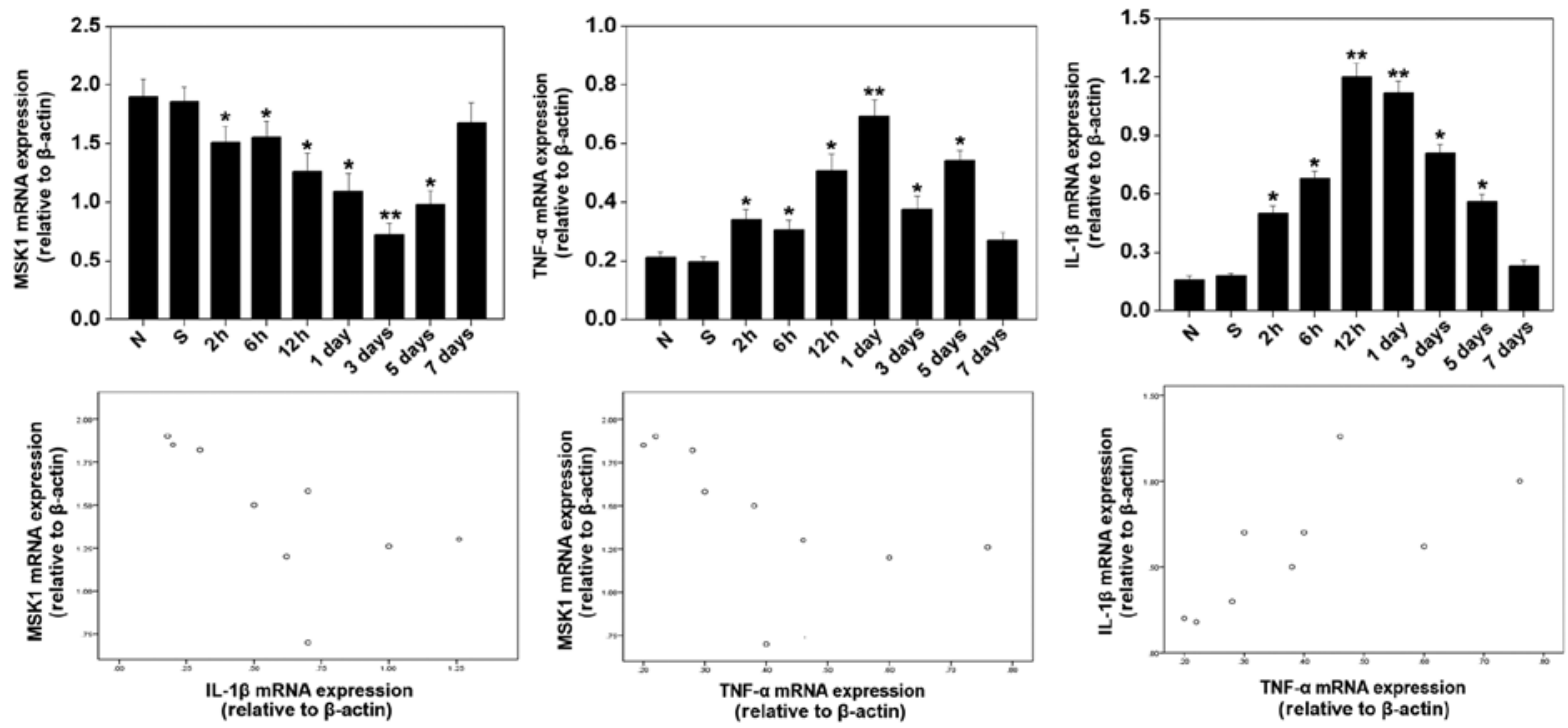

Figure 3. mRNA expression of MSK1, IL-1 $\beta$, TNF- $\alpha$ in the brain cortex at various survival time intervals after SAH. The quantitative analysis demonstrated the high expression of MSK1 mRNA in the control and sham groups. The level of MSK1 decreased gradually after SAH, then peaked at 3 days, and was increased during the following days in the cortex. Conversely, analysis of the levels of IL- $1 \beta$ and TNF- $\alpha$ mRNA indicated that these mRNA gradually increased following SAH, reaching peak levels at 1 day, and then gradually decreased during the following days in the cortex. The correlation was determined by Spearman's analysis. Bars represent the mean \pm SEM ( $n=6$ in each group). Statistical analysis was performed using one-way analysis of variance followed by Dunnett's post-hoc test. ${ }^{*} \mathrm{P}<0.05$ and ${ }^{* *} \mathrm{P}<0.01$ vs. sham and normal groups. MSK1, mitogen and stress-activated protein kinase $1 ;$ IL-1 $\beta$, interleukin-1 $\beta$; TNF- $\alpha$, tumor necrosis factor- $\alpha$; SAH, subarachnoid hemorrhage.
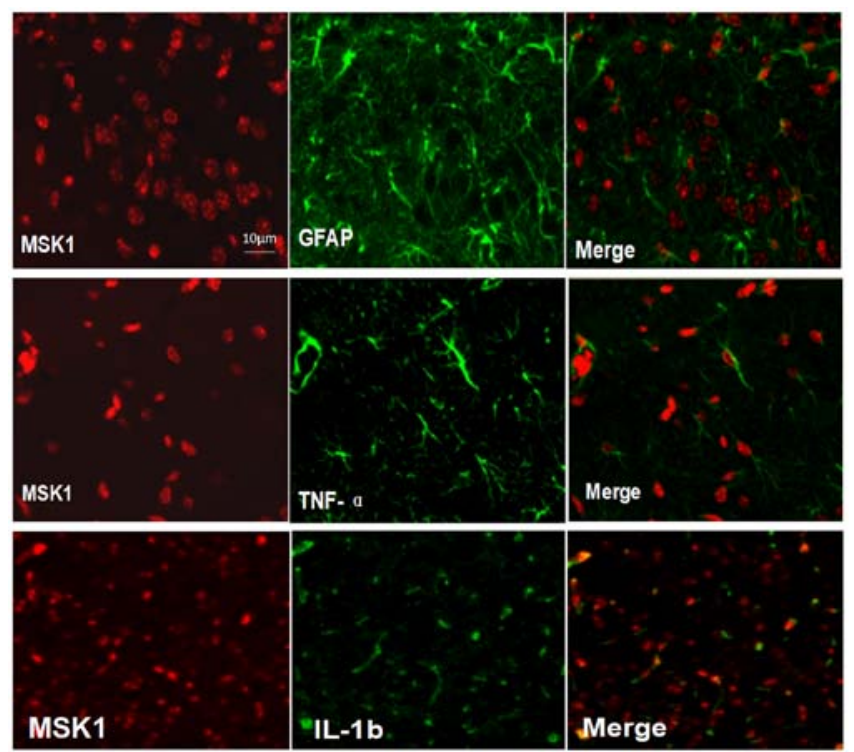

Figure 4. Double immunofluorescence staining for MSK1, TNF- $\alpha$, IL-1 $\beta$ and GFAP in brain cortex tissues at 1 day after SAH. In adult rat brain tissues after injury, sections were labeled with MSK1 and GFAP, an astrocytes marker, and the co-localization of MSK1 with GFAP was demonstrated in the brain tissues. In addition, immunofluorescence staining for MSK1, TNF- $\alpha$ and IL-1 $\beta$ was also performed, and the merged images indicated that MSK1 was closely positioned to TNF- $\alpha$ and IL-1 $\beta$, in the same view in each section. Scale bar $=10 \mu \mathrm{m}$. MSK1, mitogen and stress-activated protein kinase 1 ; IL-1 $\beta$, interleukin-1 $\beta$; TNF- $\alpha$, tumor necrosis factor- $\alpha$; GFAP, glial fibrillary acidic protein.

injury (29). The data from the present study demonstrated that the mRNA expression levels of TNF- $\alpha$ and IL-1 $\beta$ in injured brain tissues were significantly increased as early as $1 \mathrm{~h}$ after SAH compared with the sham group. These results suggested that upregulation of IL- $1 \beta$ and TNF- $\alpha$ may contribute to the

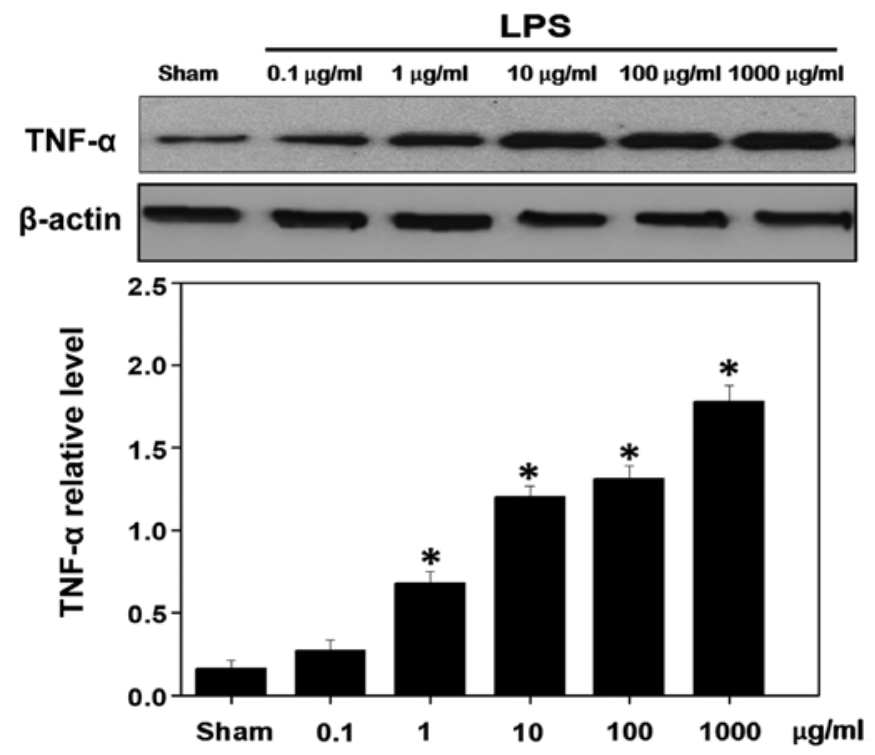

Figure 5. Different expression levels of TNF- $\alpha$ induced by LPS in cultured primary astrocytes. Western blot analysis demonstrated that LPS induced astrocytes to express TNF- $\alpha$ in a dose-dependent manner. The bar chart indicates the ratio of TNF- $\alpha$ to $\beta$-actin. Statistical analysis of the groups was performed using a one-way analysis of variance followed by Dunnett's post-hoc test. ${ }^{*} \mathrm{P}<0.05$ vs. sham group. TNF- $\alpha$, tumor necrosis factor- $\alpha$; LPS, lipopolysaccharide; $\mathrm{N}$, negative control.

initial brain inflammatory response, which then triggers the subsequent pathophysiological process, including endothelium dysfunction, BBB disruption, brain edema and neuron cell apoptosis.

However, the mechanisms of inflammation behind the pathology of SAH remain unclear. NF- $\mathrm{KB}$, which can be activated by IL-1 $\beta$ and Toll-like receptors, has been suggested to be the most important regulator during the inflammatory process 


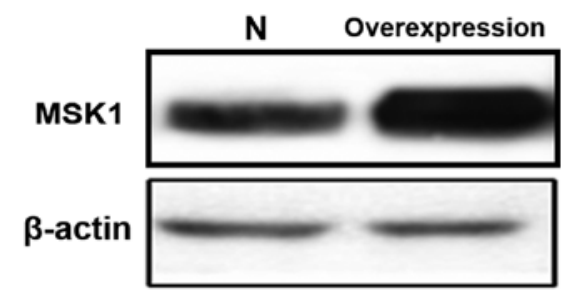

$\mathbf{N}$ LPS LPS+MSK1

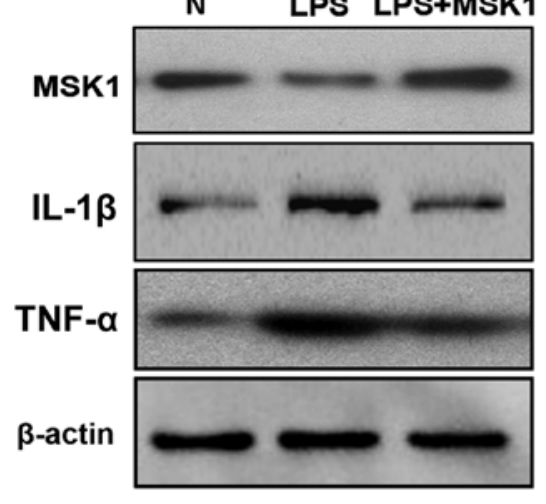

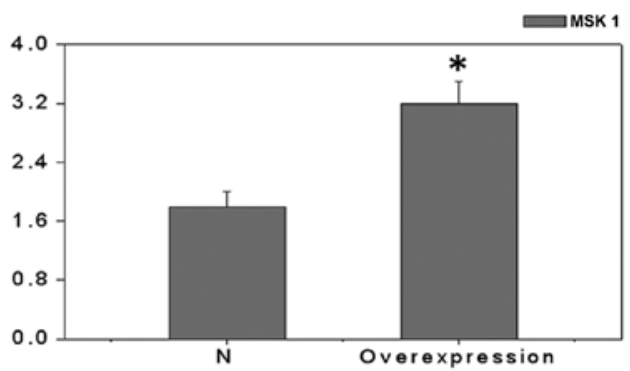

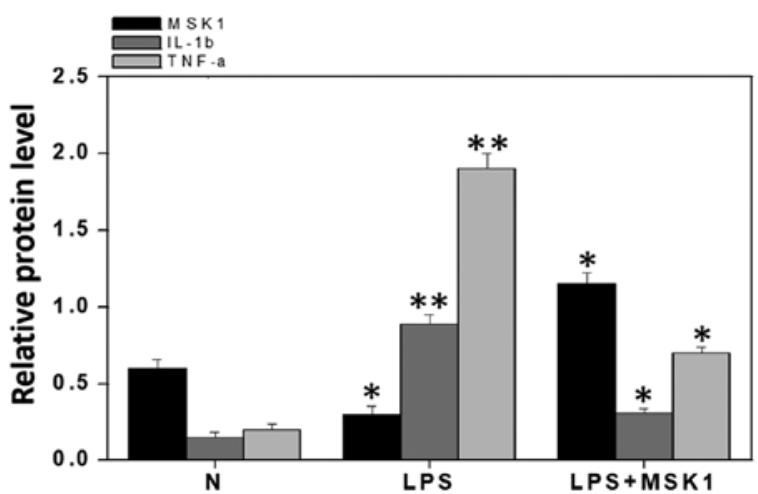

Figure 6. Western blot analysis of MSK1, IL-1 $\beta$ and TNF- $\alpha$ protein expression levels in cultured primary astrocytes post-transfection. MSK1 overexpression decreased the expression of TNF- $\alpha$. $\beta$-actin was used as a control. Statistical analysis of the groups was performed using a one-way analysis of variance followed by Dunnett's post-hoc test. ${ }^{*} \mathrm{P}<0.05$ and ${ }^{* *} \mathrm{P}<0.01$ vs. normal group. MSK1, mitogen and stress-activated protein kinase 1 ; IL-1 $\beta$, interleukin-1 $\beta$; TNF- $\alpha$, tumor necrosis factor- $\alpha$; LPS, lipopolysaccharide; N, negative control.

subsequent to $\mathrm{SAH}(30,31)$. NF- $\kappa \mathrm{B}$ is also the transcription factor target of MSK1, which can be activated in vivo downstream of mitogen-activated protein kinase (MAPK)2/ERK2 by phosphorylation of Thr-581 (32).

The MAPK and NF- $\kappa \mathrm{B}$ signaling pathways are crucial in generating inflammatory responses (33). MAPKs can regulate cell proliferation, apoptosis, differentiation and inflammatory responses through phosphorylation of their respective substrates $(34,35)$. Among the MAPK targets, MSK1 is activated downstream of p38 and ERK1/2 (11), and can mediate the phosphorylation of the NF-kB p65 subunit at Ser-276 upon IL-1 $\beta$ or TNF- $\alpha$ treatment (13). TNF- $\alpha$ induces the phosphorylation of MSK1 at Ser-360, Ser-376 and Thr-581 in airway smooth muscle cells. Dimethyl fumarate, a non-specific NF-kB inhibitor, attenuated TNF- $\alpha$-triggered MSK1 Ser-376 autophosphorylation and IL-1 $\beta$-triggered MSK1/2 phosphorylation in human keratinocytes (36). There have been multiple previous studies describing the activation of CREB, proto-oncogene c-Fos and NF-kb in inflammatory responses following subarachnoid hemorrhage (37-40). The present study focused on the involvement of MSK1 downregulation in the inflammatory responses following $\mathrm{SAH}$ in rats. We plan to investigate the role of activated MSK1 kinase and the effect of a kinase-deficient mutant of MSK1 in subsequent studies, and to examine the roles of other molecules such as CREB, c-Fos and NF- $\mathrm{kB}$ in vivo and in vitro. However, the exact mechanism of action of MSK1 in response to TNF- $\alpha$ and IL-1 $\beta$ during the inflammatory response following $\mathrm{SAH}$ remains unknown, and to the best of our knowledge, direct genetic evidence supporting the role of MSK1 in CNS inflammation following SAH has not been reported to date.
In the present study, the expression of TNF- $\alpha$ and IL- $1 \beta$ gradually increased $1 \mathrm{~h}$ after $\mathrm{SAH}$, peaked at day 1 post-SAH and decreased during the following days. Notably, the expression of MSK1 exhibited a negative correlation with TNF- $\alpha$ and IL-1 $\beta$ expression. It gradually decreased $1 \mathrm{~h}$ after SAH, peaked at day 3 post-SAH and increased during the following days. Double immunofluorescence staining revealed that TNF- $\alpha$ and GFAP were labeled and co-localized in adult rat brain at day 1 after SAH. In addition, microscopy analysis revealed that MSK1 was closely positioned to TNF- $\alpha$ and IL-1 $\beta$ in the same view in the brain cortex. These data suggested that MSK1 may be associated with the inflammatory response subsequent to SAH. MSK1 may mediate inflammatory responses via a negative feedback loop to decrease the expression of pro-inflammation factors. The observed co-localization of MSK1 and TNF- $\alpha$ supported this hypothesis, but the detailed mechanisms require further investigation.

It is well-known that MSK1 has a high level of expression in astrocytes (41), which is associated with numerous inflammatory responses of the CNS (42). Therefore, the present study established an LPS-induced astrocytic inflammation model to confirm whether MSK1 serves a role in pro-inflammatory cytokine expression. The effect of MSK1 overexpression on astrocytes was analyzed by western blot analysis. The results suggested that MSK1 inhibited astrocytic inflammation by attenuating the expression of IL-1 $\beta$ and TNF- $\alpha$. During the inflammatory response, NF-kB potentially connects MSK1 with pro-inflammatory factors, although future studies investigating the effect of kinase-deficient mutant of MSK1 using small interference RNA or specific inhibitors are required to confirm this hypothesis. 
Despite it is known that inflammatory reactions occur upon $\mathrm{SAH}$, a link must be identified between inflammation and poor outcomes following SAH in order to consider inflammation as an important therapeutic target. Our in vivo and in vitro experiments implicated that M SK1 may be a key inflammatory regulator. Regulation of MSK1 expression in activated astrocytes may provide a novel strategy to protect against inflammation-induced injury.

\section{Acknowledgements}

Not applicable

\section{Funding}

The present study was supported by the National Natural Science Foundation of China (grant no. 81671129) and the Natural Science Foundation of Fujian (grant no. 13185026).

\section{Availability of data and materials}

The datasets used and/or analyzed during the present study are available from the corresponding author on reasonable request.

\section{Authors' contributions}

$\mathrm{BN}, \mathrm{ZL}, \mathrm{FL}$ and $\mathrm{BZ}$ designed the experiments. BN, ZL, JW, $\mathrm{XC}$ and $\mathrm{PJ}$ performed the in vitro and in vivo experiments. $\mathrm{LN}$ analyzed the data and images. BN and $\mathrm{ZL}$ wrote the manuscript. All authors read and approved the final manuscript.

\section{Ethics approval and consent to participate}

The animal use and care protocols, including all surgical procedures, were approved by The Animal Care and Use Committee of Taishan Medical University (approval no. 2014-002) and conformed to the Guide for the Care and Use of Laboratory Animals by the National Institute of Health

\section{Patient approval for publication}

Not applicable

\section{Competing interests}

The authors declare that they have no competing interests.

\section{References}

1. van Gijn J, Kerr RS and Rinkel GJ: Subarachnoid haemorrhage. Lancet 369: 306-318, 2007.

2. Alg VS, Sofat R, Houlden H and Werring DJ: Genetic risk factors for intracranial aneurysms: a meta-analysis in more than 116,000 individuals. Neurology 80: 2154-2165, 2013.

3. Connolly ES Jr, Rabinstein AA, Carhuapoma JR, Derdeyn CP, Dion J, Higashida RT, Hoh BL, Kirkness CJ, Naidech AM, Ogilvy CS, et al; American Heart Association Stroke Council; Council on Cardiovascular Radiology and Intervention; Council on Cardiovascular Nursing; Council on Cardiovascular Surgery and Anesthesia; Council on Clinical Cardiology: Guidelines for the management of aneurysmal subarachnoid hemorrhage: A guideline for healthcare professionals from the American Heart Association/american Stroke Association. Stroke 43: 1711-1737, 2012.
4. Rinkel GJ and Algra A: Long-term outcomes of patients with aneurysmal subarachnoid haemorrhage. Lancet Neurol 10: 349-356, 2011.

5. Sehba FA, Pluta RM and Zhang JH: Metamorphosis of subarachnoid hemorrhage research: From delayed vasospasm to early brain injury. Mol Neurobiol 43: 27-40, 2011.

6. Provencio JJ: Inflammation in subarachnoid hemorrhage and delayed deterioration associated with vasospasm: A review. Acta Neurochir Suppl (Wien) 115: 233-238, 2013.

7. Jedrzejowska-Szypulka H, Straszak G and Laryszbrysz M: Interleukin-1beta plays a role in the activation of peripheral leukocytes after blood-brain barrier rupture in the course of subarachnoid hemorrhage. Curr Neurovasc Res 7: 39-48, 2010.

8. Xie X, Wu X, Cui J, Li H and Yan X: Increase ICAM-1 and LFA-1 expression by cerebrospinal fluid of subarachnoid hemorrhage patients: Involvement of TNF- $\alpha$. Brain Res 1512: 89-96, 2013.

9. Jiang Y, Liu DW, Han XY, Dong YN, Gao J, Du B, Meng L and Shi JG: Neuroprotective effects of anti-tumor necrosis factor-alpha antibody on apoptosis following subarachnoid hemorrhage in a rat model. J Clin Neurosci 19: 866-872, 2012.

10. Drobic B, Espino PS and Davie JR: Mitogen- and stress-activated protein kinase 1 activity and histone $\mathrm{h} 3$ phosphorylation in oncogene-transformed mouse fibroblasts. Cancer Res 64: 9076-9079, 2004.

11. Deak M, Clifton AD, Lucocq LM and Alessi DR: Mitogen- and stress-activated protein kinase-1 (MSK1) is directly activated by MAPK and SAPK2/p38, and may mediate activation of CREB. EMBO J 17: 4426-4441, 1998.

12. Vermeulen L, De Wilde G, Van Damme P, Vanden Berghe W and Haegeman G: Transcriptional activation of the NF-kappaB p65 subunit by mitogen- and stress-activated protein kinase-1 (MSK1). EMBO J 22: 1313-1324, 2003.

13. Reber L, Vermeulen L, Haegeman G and Frossard N: Ser276 phosphorylation of NF-kB p65 by MSK1 controls SCF expression in inflammation. PLoS One 4: e4393, 2009.

14. Ananieva O, Darragh J, Johansen C, Carr JM, McIlrath J, Park JM, Wingate A, Monk CE, Toth R, Santos SG, et al: The kinases MSK1 and MSK2 act as negative regulators of Toll-like receptor signaling. Nat Immunol 9: 1028-1036, 2008.

15. Beck IM, Vanden Berghe W, Vermeulen L, Bougarne N, Vander Cruyssen B, Haegeman G and De Bosscher K: Altered subcellular distribution of MSK1 induced by glucocorticoids contributes to NF-kappaB inhibition. EMBO J 27: 1682-1693, 2008.

16. National Research Council (US) Institute for Laboratory Animal Research: Guide for the Care and Use of Laboratory Animals. National Academies Press (US), Washington, DC, 1996.

17. Livak KJ and Schmittgen TD: Analysis of relative gene expression data using real-time quantitative PCR and the $2-\Delta \Delta C T$ method. Methods 25: 402-408, 2001.

18. Ning B, Guo G, Liu H, Ning L, Sun BL, Li Z, Wang S, Lv ZW and Fan CD: MSK1 downregulation is associated with neuronal and astrocytic apoptosis following subarachnoid hemorrhage in rats. Oncol Lett 14: 2940-2946, 2017.

19. Dorsch NW: Cerebral arterial spasm - a clinical review. Br J Neurosurg 9: 403-412, 1995.

20. Dziurdzik P, Krawczyk L, Jalowiecki P, Kondera-Anasz Z and Menon L: Serum interleukin-10 in ICU patients with severe acute central nervous system injuries. Inflamm Res 53: 338-343, 2004.

21. Ostrowski RP, Colohan AR and Zhang JH: Molecular mechanisms of early brain injury after subarachnoid hemorrhage. Neurol Res 28: 399-414, 2006.

22. Macdonald RL, Kakarieka A, Mayer SA, Pasqualin A, Rufenacht DA, Schmiedek P and Kassell NF: Prevention of cerebral vasospasm after aneurysmal subarachnoid hemorrhage with clazosentan, an endothelin receptor antagonist. Neurosurgery 59: 453, 2006.

23. Cahill J, Calvert JW and Zhang JH: Mechanisms of early brain injury after subarachnoid hemorrhage. J Cereb Blood Flow Metab 26: 1341-1353, 2006.

24. Macdonald RL, Pluta RM and Zhang JH: Cerebral vasospasm after subarachnoid hemorrhage: The emerging revolution. Nat Clin Pract Neurol 3: 256-263, 2007.

25. Macdonald RL, Kassell NF, Mayer S, Ruefenacht D, SchmiedekP Weidauer S, Frey A, Roux S and Pasqualin A; CONSCIOUS-1 Investigators: Clazosentan to overcome neurological ischemia and infarction occurring after subarachnoid hemorrhage (CONSCIOUS-1): Randomized, double-blind, placebo-controlled phase 2 dose-finding trial. Stroke 39: 3015-3021, 2008. 
26. Hanafy KA, Grobelny B, Fernandez L, Kurtz P, Connolly ES Mayer SA, Schindler C and Badjatia N: Brain interstitial fluid TNF-alpha after subarachnoid hemorrhage. J Neurol Sci 291: 69-73, 2010.

27. Kaynar MY, Tanriverdi T, Kafadar AM, Kacira T, Uzun H, Aydin S, Gumustas K, Dirican A and Kuday C: Detection of soluble intercellular adhesion molecule-1 and vascular cell adhesion molecule- 1 in both cerebrospinal fluid and serum of patients after aneurysmal subarachnoid hemorrhage. J Neurosurg 101: 1030-1036, 2004.

28. Kikuchi T, Okuda Y, Kaito N and Abe T: Cytokine production in cerebrospinal fluid after subarachnoid haemorrhage. Neurol Res 17: 106-108, 1995.

29. Aoki $\mathrm{T}$ and Nishimura M: Targeting chronic inflammation in cerebral aneurysms: Focusing on NF-kappaB as a putative targe of medical therapy. Expert Opin Ther Targets 14: 265-273, 2010

30. Greenhalgh AD, Brough D, Robinson EM, Girard S, Rothwell NJ and Allan SM: Interleukin-1 receptor antagonist is beneficial after subarachnoid haemorrhage in rat by blocking haem-driven inflammatory pathology. Dis Model Mech 5: 823-833, 2012.

31. Ma CX, Yin WN and Cai BW: Activation of TLR4/ NF- $\kappa \mathrm{B}$ signaling pathway in early brain injury after subarachnoid haemorrhage. Neurol Res 122: 1575-1581, 2009.

32. Arthur JS: MSK activation and physiological roles. Front Biosci 13: 5866-5879, 2008.

33. Arthur JSC and Ley SC: Mitogen-activated protein kinases in innate immunity. Nat Rev Immunol 13: 679-692, 2013.

34. Kim JH, Choi JS and Lee BH: PI3K/Akt and MAPK pathways evoke activation of FoxO transcription factor to undergo neuronal apoptosis in brain of the silkworm Bombyx mori (Lepidoptera: Bombycidae). Cell Mol Biol 58: OL1780-OL1785, 2012.

35. Pan WW, Li JD, Huang S, Papadimos TJ, Pan ZK and Chen LY: Synergistic activation of NF- $\{\kappa\} \mathrm{B}$ by bacterial chemoattractant and TNF $\{\alpha\}$ is mediated by p38 MAPK-dependent RelA acetylation. J Biol Chem 285: 34348-34354, 2010.
36. Gesser B, Johansen C, Rasmussen MK, Funding AT, Otkjaer K, Kjellerup RB, Kragballe K and Iversen L: Dimethylfumarate specifically inhibits the mitogen and stress-activated kinases 1 and 2 (MSK1/2): Possible role for its anti-psoriatic effect. J Invest Dermatol 127: 2129-2137, 2007.

37. Peng Y, Jin J, Fan L, Xu H, He P, Li J, Chen T, Ruan W and Chen G: Rolipram Attenuates Early Brain Injury Following Experimental Subarachnoid Hemorrhage in Rats: Possibly via Regulating the SIRT1/NF- $\kappa$ B Pathway. Neurochem Res 43: 785-795, 2018

38. You W, Zuo G, Shen H, Tian X, Li H, Zhu H, Yin J, Zhang T and Wang Z: Potential dual role of nuclear factor-kappa B in experimental subarachnoid hemorrhage-induced early brain injury in rabbits. Inflamm Res 65: 975-984, 2016.

39. Mo J, Enkhjargal B, Travis ZD, Zhou K, Wu P, Zhang G, Zhu Q, Zhang T, Peng J, Xu W, et al: AVE 0991 attenuates oxidative stress and neuronal apoptosis via Mas/PKA/CREB/UCP-2 pathway after subarachnoid hemorrhage in rats. Redox Biol 20: 75-86, 2019.

40. Lv O, Zhou F, Zheng Y, Li Q, Wang J and Zhu Y: Mild hypothermia protects against early brain injury in rats following subarachnoid hemorrhage via the TrkB/ERK/CREB signaling pathway. Mol Med Rep 14: 3901-3907, 2016.

41. Ning B, Li Z, Zhu N, Hou G and Pang Q: Traumatic brain injury induces a downregulation of MSK1 in rat brain cortex. J Mol Neurosci 49: 380-386, 2013.

42. Myer DJ, Gurkoff GG, Lee SM, Hovda DA and Sofroniew MV: Essential protective roles of reactive astrocytes in traumatic brain injury. Brain 129: 2761-2772, 2006.

This work is licensed under a Creative Commons Attribution-NonCommercial-NoDerivatives 4.0 International (CC BY-NC-ND 4.0) License. 